\title{
Burden of pneumococcal disease: 8-year retrospective analysis from a single centre in Hong Kong
}

\author{
MY Man *, HP Shum, Judianna SY Yu, Alan Wu, WW Yan
}

\section{A B S T R A C T}

Purpose: Streptococcus pneumoniae is a common pathogen involved in community-acquired pneumonia. Invasive pneumococcal disease is often associated with higher co-morbidity rates, but mortality-related findings have been inconclusive. This study investigated predictors of 30-day mortality and invasive pneumococcal disease.

Methods: This retrospective analysis included adults with pneumococcal disease who were admitted to Pamela Youde Nethersole Eastern Hospital from 1 January 2011 to 31 December 2018. Demographics, microbiological characteristics, and outcomes were compared between 30-day survivors and non-survivors, and between patients with invasive disease and those with non-invasive disease. Intensive care unit (ICU) subgroup analysis was performed. The primary outcome was 30-day all-cause mortality; secondary outcomes were ICU and hospital mortalities, and ICU and hospital lengths of stay.

Results: In total, 792 patients had pneumococcal disease; 701 survived and 91 (11.5\%) died within 30 days. Notably, 106 (13.4\%) patients had invasive pneumococcal disease and 170 (21.5\%) patients received intensive care. Vasopressor use (odds ratio $[\mathrm{OR}]=4.96, \mathrm{P}<0.001)$, chronic kidney disease
This article was published on $9 \mathrm{Jul}$ 2020 at www.hkmj.org. results $(\mathrm{OR}=257, \mathrm{P}=0.001)$ and advanced age
$(\mathrm{OR}=2.19, \mathrm{P}=0.010)$ were independent predictors for 30-day mortality by logistic regression analysis. Among critically ill patients, chronic kidney disease $(\mathrm{OR}=4.64, \mathrm{P}<0.001)$, higher APACHE IV score $(\mathrm{OR}=3.73, \mathrm{P}=0.016)$, and positive urinary antigen test results $(\mathrm{OR}=2.94, \mathrm{P}=0.008)$ were predictors for 30-day mortality. Logistic regression analysis revealed that chronic kidney disease $(\mathrm{OR}=3.10$, $\mathrm{P}<0.001)$ was a risk factor for invasive pneumococcal disease.

Conclusion: Advanced age, vasopressor use, chronic kidney disease, and positive urinary antigen test results were independent predictors for 30-day mortality in patients with pneumococcal disease.

\section{Hong Kong Med J 2020;26:372-81}

https://doi.org/10.12809/hkmj208373

${ }^{1}$ MY Man *, MB, BS, FHKAM (Medicine)

${ }^{1}$ HP Shum, MB, BS, MD

JSY Yu, MB, BS, MRCP (UK)

${ }^{3} \mathrm{~A}$ Wu, MB, ChB, FRCPath (UK)

${ }^{1}$ WW Yan, FRCP, FHKAM (Medicine)

Department of Intensive Care, Pamela Youde Nethersole Eastern Hospital, Hong Kong

${ }^{2}$ Department of Medicine and Geriatrics, Ruttonjee and Tang Shiu Kin Hospital, Hong Kong

Department of Clinical Pathology, Pamela Youde Nethersole Eastern Hospital, Hong Kong

* Corresponding author: mmy553@ha.org.hk

New knowledge added by this study

- This is one of the largest studies thus far regarding pneumococcal infection in Hong Kong; it also includes an analysis of critically ill patients.

- Invasive pneumococcal disease was associated with greater disease severity and higher rates of invasive organ support. Positive urinary pneumococcal antigen test results were associated with increased 30-day mortality rates in all patients, as well as patients in the intensive care unit.

- The 30-day mortality predictors of pneumococcal disease included vasopressor use, chronic kidney disease, positive urinary antigen test results, and advanced age.

Implications for clinical practice or policy

- Invasive pneumococcal disease is associated with more severe disease and higher mortality rates. Rapid identification and treatment can improve patient outcomes.

- Increasing use of the urinary antigen test was observed during the study period. A positive urinary antigen test result can serve as an independent predictor for 30-day mortality in all patients, as well as patients in the intensive care unit.

\section{Introduction}

Streptococcus pneumoniae causes a wide range of diseases that include middle ear infection, sinusitis, pneumonia, and meningitis. As one of the most common pathogens in community-acquired pneumonia (especially in Western countries), 
$S$ pneumoniae infection contributed to 1.6 million deaths in 2010 and 3.7 million severe pneumococcal infections worldwide in 2015. ${ }^{1-3}$

Streptococcus pneumoniae is a gram-positive encapsulated bacterium that colonises human nasopharynx and is mainly transmitted via respiratory droplets, which cause middle ear and respiratory tract infection. Thus far, more than 90 serotypes of $S$ pneumoniae have been identified. Streptococcus pneumoniae infection can be stratified into invasive and non-invasive disease. ${ }^{4,5}$ Invasive pneumococcal disease (IPD) is a notifiable disease in Hong Kong. In 2019, there were 187 cases; the incidence has remained similar over the past few years. ${ }^{6}$ Worldwide, there is growing concern regarding drug-resistant $S$ pneumoniae strains (eg, strains resistant to macrolide, penicillin, and/or fluoroquinolone). However, drug-resistant strains have not been associated with higher mortality rates. ${ }^{7}$ The prevalence of drug-resistant $S$ pneumoniae is lower in Southeast Asia than in Western countries. ${ }^{1}$ Despite inconclusive evidence in the literature regarding its association with mortality, IPD is often associated with more severe disease and requires more invasive organ support. ${ }^{8}$

In this study, we aimed to identify the predictors for 30-day mortality in patients with $S$ pneumoniae infection, as well as predictors for IPD. We also performed subgroup analysis of patients in the intensive care unit (ICU) and identified risk factors for 30-day mortality and IPD in those patients, as well as all patients with $S$ pneumoniae infection.

\section{Methods}

\section{Study design and data collection}

This retrospective cohort study included adults who were admitted to Pamela Youde Nethersole Eastern Hospital, Hong Kong, with pneumococcal infection from 1 January 2011 to 31 December 2018. Patients who were aged $<18$ years or had incomplete data were excluded.

Patient medical records and data were extracted from clinical management systems and clinical information systems (IntelliVue Clinical Information Portfolio; Philips Medical, Amsterdam, The Netherlands). Baseline demographics, clinical characteristics, and microbiological data were identified. For patients in the ICU, disease severity was quantified using APACHE (Acute Physiology and Chronic Health Evaluation) IV scores. The use of invasive organ support was recorded, including continuous renal replacement therapy, inotropes, invasive mechanical ventilation, and extracorporeal membrane oxygenation. The primary outcome was 30-day all-cause mortality; secondary outcomes were ICU and hospital mortalities, ICU and hospital length of stay (LOS), and ICU ventilator days.

\section{肺炎鏈球菌：八年回顧研究 \\ 文敏儀、沈海平、余雪瑩、胡家倫、殷榮華}

目的：肺炎鏈球菌是引致社區肺炎最常見的病菌之一。有關入侵性肺 炎鏈球菌的文獻甚多, 但鮮有關於死亡率的研究。這項研究的目的在 於找出 30 天死亡率及入侵性肺炎鏈球菌的誘因。

方法：這項回顧研究納入 2011 年1月1日至2018年12月31日在東區尤 德夫人那打素醫院留醫的肺炎鏈球菌患者。本研究就 30 天存活者和死 者的人口統計資料和微生物學特徵, 以及肺炎鏈球菌患者與入侵性肺 炎鏈球菌患者作出比較。

結果：研究期間, 792 名病人患有肺炎鏈球菌。701人存活, 91 人 $(11.5 \%)$ 於 30 天內死亡。當中 106 人 ( $13.4 \%)$ 為入侵性肺炎鏈球 菌患者, 170 人 $(21.5 \%)$ 須接受危重治療。邏輯迴歸分析顯示使用 血管加壓藥（比值比 $=4.96, \mathrm{P}<0.001$ ) 、慢性腎病（比值比 $=3.62$, $\mathrm{P}<0.001$ ) 、尿液肺炎鏈球菌快速抗原檢測呈陽性（比值比 $=2.57$, $\mathrm{P}=0.001)$ 及老年患者 $($ 比值比 $=2.19, \mathrm{P}=0.010)$ 是 30 天死亡率的獨 立預測因子。在重症患者中, 慢性腎病（比值比 $=4.64, \mathrm{P}<0.001$ )

APACHE IV 評分較高 (比值比 $=3.73, \mathrm{P}=0.016)$ 和尿液肺炎鏈球菌 快速抗原檢測呈陽性（比值比 $=2.94, \mathrm{P}=0.008$ ) 是 30 天死亡率的獨立 預測因子。邏輯迴歸分析顯示慢性腎病 $($ 比值比 $=3.10, \mathrm{P}<0.001)$ 是 入侵性肺炎鏈球菌疾病的危險因素。

結論：老年患者、使用血管加壓藥、慢性腎病及尿液肺炎鏈球菌快速 抗原檢測呈陽性均為肺炎鏈球菌患者在 30 天內死亡的最重要誘因。

\section{Definitions}

Pneumococcal infection was determined by positive culture of $S$ pneumoniae. Invasive pneumococcal disease was defined as the presence of $S$ pneumoniae in sterile sites (eg, pleural fluid, cerebrospinal fluids, and blood). ${ }^{4,8}$ Non-invasive pneumococcal disease was defined as the presence of $S$ pneumoniae in non-sterile sites, or a positive urinary antigen test (UAT) result. Medical co-morbidities (eg, diabetes mellitus, chronic kidney disease, heart failure, and haematological malignancies) were coded in accordance with the International Classification of Diseases, Ninth Revision, Clinical Modification. Smokers were defined as those who had ever smoked. Advanced age was defined as age $>65$ years.

\section{Microbiology}

Antibiotic resistance was determined based on Clinical and Laboratory Standards Institute testing criteria for minimal inhibitory concentrations. Breakpoints adopted for determination of parenteral penicillin resistance in non-meningitis $S$ pneumoniae isolates were susceptible, $\leq 2 \mu \mathrm{g} / \mathrm{mL}$; intermediate, $4 \mu \mathrm{g} / \mathrm{mL}$; and resistance, $\geq 8 \mu \mathrm{g} / \mathrm{mL}$. ${ }^{9}$ Breakpoints adopted for determination of parenteral penicillin resistance in meningitis $S$ pneumoniae isolates were susceptible, $\leq 0.06 \mu \mathrm{g} / \mathrm{mL}$ and resistance, 
$\geq 0.12 \mu \mathrm{g} / \mathrm{mL}$; breakpoints adopted for determination of levofloxacin resistance in $S$ pneumoniae were susceptible, $\leq 2 \mu \mathrm{g} / \mathrm{mL}$; intermediate, $4 \mu \mathrm{g} / \mathrm{mL}$; and resistance, $\geq 8 \mu \mathrm{g} / \mathrm{mL}$. $^{9}$

Urinary antigen test (Alere 710-012 BinaxNOW Streptococcus) results were evaluated in accordance with the manufacturer's instructions.

\section{Statistical analysis}

Characteristics and clinical parameters were compared between patients with IPD and those with non-invasive pneumococcal disease, as well as between 30-day survivors and non-survivors. Results were expressed as median (interquartile range) or as numbers (percentages) of cases, as appropriate. For univariate analysis, categorical variables were compared by Pearson Chi squared tests or Fisher's exact test, as appropriate; continuous variables were compared by using the Mann-Whitney $U$ test. Variables with $\mathrm{P}<0.2$ in univariate analysis or with known clinical significance from previous studies were entered into multivariate analysis. Independent predictors for 30-day mortality and independent predictors for IPD were assessed by logistic regression analysis. ${ }^{8,10-12}$ Subgroup analysis was performed regarding IPD and disease severity among patients in the ICU. Hosmer-Lemeshow test was performed for goodness-of-fit for logistic regression models. Kaplan-Meier survival plots were used to compare cumulative survival between patients with IPD and those with non-invasive pneumococcal disease. SPSS (Mac version 24.0; IBM Corp, Armonk [NY], United States) was used for all statistical analyses.

\section{Results}

Patient demographic and clinical characteristics, including co-morbidities and use of invasive organ support, are shown in Table 1. In total, 792 patients with pneumococcal disease were identified during the 8-year study period. The median age was 73 years; patients were predominantly men. Most patients exhibited respiratory tract infection (96.1\%) and approximately one quarter of patients had asthma/chronic obstructive pulmonary disease (24.4\%). In total, 170 patients received intensive care and $14.1 \%$ required invasive mechanical ventilation; $28 \%$ required vasopressor use. Invasive pneumococcal disease was present in $13.4 \%$ of the patients. The overall hospital mortality rate was $11.2 \%$, while the mortality rate among patients in the ICU was $22.9 \%$.

Invasive pneumococcal disease was associated with a higher 30 -day mortality rate $(28.6 \%$ vs $11.4 \%$, $\mathrm{P}<0.001)$; a positive UAT result was also associated with a higher 30 -day mortality rate $(36.3 \%$ vs $12.7 \%$, $\mathrm{P}<0.001)$. Logistic regression analysis identified statistically significant predictors for 30-day mortality, which are shown in Table 1 . Patients with vasopressor use (odds ratio $[\mathrm{OR}]=4.96, \mathrm{P}<0.001$ ), chronic kidney disease $(\mathrm{OR}=3.62, \mathrm{P}<0.001)$, a positive UAT result $(\mathrm{OR}=2.57, \mathrm{P}=0.001)$, and older age $(\mathrm{OR}=2.19, \mathrm{P}=0.010)$ exhibited comparatively higher 30-day mortality rates; however, asthma/chronic obstructive pulmonary disease was not an independent predictor for mortality in logistic regression analysis. The Figure depicts the results of Kaplan-Meier survival analysis comparing patients with IPD and those with non-invasive pneumococcal disease.

Table 2 shows the characteristics of patients with IPD and those with non-invasive pneumococcal disease. More patients with asthma/chronic obstructive pulmonary disease exhibited non-invasive pneumococcal disease $(26.5 \%$ vs $10.4 \%, \mathrm{P}<0.001)$. Invasive pneumococcal disease was more likely to be associated with renal failure $(27.4 \%$ vs $9.6 \%, \mathrm{P}<0.001)$ and haematological malignancy $(5.7 \%$ vs $1.7 \%$, $\mathrm{P}=0.012)$. Additionally, IPD was associated with higher rates of ICU admission $(33.0 \%$ vs $19.7 \%$, $\mathrm{P}=0.002)$, renal replacement therapy $(16.0 \%$ vs $4.8 \%$, $\mathrm{P}<0.001)$, and vasopressor use $(93.4 \%$ vs $17.9 \%$, $\mathrm{P}<0.001)$. Patients with IPD had a higher 30-day mortality rate $(24.5 \%$ vs $9.5 \%, \mathrm{P}<0.001)$ and longer hospital LOS ( 8 vs 4 days, $\mathrm{P}<0.001$ ). Independent risk factors for IPD by logistic regression analysis are shown in Table 2, along with their ORs. Notably, chronic kidney disease $(\mathrm{OR}=3.10, \mathrm{P}<0.001)$ was the sole independent predictor for IPD.

The results of ICU subgroup analysis are shown in Table 3. Respiratory tract infection constituted $93.5 \%$ of all $S$ pneumoniae infections. The rate of IPD was $20.6 \%$ among patients in the ICU with $S$ pneumoniae infection, which was higher than the rate among all patients with $S$ pneumoniae infection. Further analysis revealed that IPD was associated with higher rates of complications and invasive organ support; in particular, more patients with IPD required renal replacement therapy (48.6\% vs $24.4 \%, \mathrm{P}=0.005)$ and vasopressor use (100\% vs $88.9 \%, \quad \mathrm{P}=0.039)$. Additionally, more patients with IPD tended to exhibit pleural effusion/empyema, although this difference was not statistically significant. Patients who required invasive mechanical ventilation $(76.0 \%$ vs $57.5 \%$, $\mathrm{P}=0.023)$, extracorporeal membrane oxygenation (14.0\% vs $5.0 \%, \mathrm{P}=0.044)$, renal replacement therapy (48.0\% vs $21.7 \%, \mathrm{P}=0.001$ ), and vasopressor use (98.0\% vs $88.3 \%, \mathrm{P}=0.043$ ) exhibited significantly higher 30-day mortality rates. Logistic regression analysis showed that chronic kidney disease $(\mathrm{OR}=4.64, \mathrm{P}<0.001)$, higher APACHE IV score $(\mathrm{OR}=3.73, \mathrm{P}=0.016)$, and a positive UAT result $(\mathrm{OR}=2.94, \mathrm{P}=0.008)$ were independent predictors for 30-day mortality among patients in the ICU who had IPD (Table 3). 
TABLE I. Clinical predictors of 30-day mortality in patients with pneumococcal disease*

\begin{tabular}{|c|c|c|c|c|c|}
\hline Characteristics & Total $(n=792)$ & $\begin{array}{c}\text { 30-Day non- } \\
\text { survivors }(n=91)\end{array}$ & $\begin{array}{c}\text { 30-Day survivors } \\
(\mathrm{n}=701)\end{array}$ & $P$ value & Odds ratio $(95 \% \mathrm{Cl})$ \\
\hline Age (years) & $73(61-81)$ & $79(68-85)$ & $72(61-81)$ & $<0.001$ & \\
\hline Advanced age ( $>65$ years) & $554(69.9 \%)$ & $72(79.1 \%)$ & $482(68.8 \%)$ & 0.043 & $2.19(1.21-3.99) \dagger$ \\
\hline Sex & & & & 0.849 & \\
\hline Male & $581(73.4 \%)$ & $66(72.5 \%)$ & 515 (73.5\%) & & \\
\hline Female & $211(26.6 \%)$ & $25(27.5 \%)$ & $186(26.5 \%)$ & & \\
\hline Ever smoker & $200(25.3 \%)$ & $9(9.9 \%)$ & $191(27.2 \%)$ & $<0.001$ & \\
\hline Sources of admission & & & & 0.318 & \\
\hline AED & $734(92.7 \%)$ & $82(90.1 \%)$ & $652(93.0 \%)$ & & \\
\hline Clinical/transfers & $58(7.3 \%)$ & $9(9.9 \%)$ & $49(7.0 \%)$ & & \\
\hline Sources of infection & & & & 0.409 & \\
\hline CNS & $3(0.4 \%)$ & $1(1.1 \%)$ & $2(0.3 \%)$ & & \\
\hline MSK & $2(0.3 \%)$ & 0 & $2(0.3 \%)$ & & \\
\hline Respiratory & $761(96.1 \%)$ & $86(94.5 \%)$ & $675(96.3 \%)$ & & \\
\hline Septicaemia & $10(1.3 \%)$ & $1(1.1 \%)$ & $9(1.3 \%)$ & & \\
\hline Skin & $5(0.6 \%)$ & 0 & $5(0.7 \%)$ & & \\
\hline Others & $11(1.4 \%)$ & $3(3.3 \%)$ & $8(1.1 \%)$ & & \\
\hline \multicolumn{6}{|l|}{ Co-morbidities } \\
\hline Heart failure & $31(3.9 \%)$ & $8(8.8 \%)$ & $23(3.3 \%)$ & 0.011 & \\
\hline Asthma/COPD & $193(24.4 \%)$ & $8(8.8 \%)$ & 185 (26.4\%) & $<0.001$ & \\
\hline Chronic kidney disease & $95(12.0 \%)$ & $40(44.0 \%)$ & $55(7.8 \%)$ & $<0.001$ & $3.62(2.04-6.42) \dagger$ \\
\hline Cirrhosis & $10(1.3 \%)$ & 0 & $10(1.4 \%)$ & 0.615 & \\
\hline Haematological malignancy & $18(2.3 \%)$ & $4(4.4 \%)$ & $14(2.0 \%)$ & 0.142 & \\
\hline Metastatic cancer & $2(0.3 \%)$ & 0 & $2(0.3 \%)$ & 1.000 & \\
\hline Chemotherapy & $68(8.6 \%)$ & $6(6.6 \%)$ & $62(8.8 \%)$ & 0.471 & \\
\hline Diabetes mellitus & $131(16.5 \%)$ & $14(15.4 \%)$ & $117(16.7 \%)$ & 0.752 & \\
\hline Immunocompromised status & $9(1.1 \%)$ & $2(2.2 \%)$ & $7(1.0 \%)$ & 0.277 & \\
\hline \multicolumn{6}{|c|}{ Complications and invasive organ support } \\
\hline Intensive care & $170(21.5 \%)$ & $50(54.9 \%)$ & $120(17.1 \%)$ & $<0.001$ & \\
\hline Pleural effusion/empyema & $40(5.1 \%)$ & $6(6.6 \%)$ & $34(4.9 \%)$ & 0.475 & \\
\hline Invasive mechanical ventilation & $112(14.1 \%)$ & $40(44.0 \%)$ & $72(10.3 \%)$ & $<0.001$ & \\
\hline ECMO & $13(1.6 \%)$ & $7(7.7 \%)$ & $6(0.9 \%)$ & $<0.001$ & \\
\hline Viral co-infection & $22(2.8 \%)$ & $2(2.2 \%)$ & $20(2.9 \%)$ & 1.000 & \\
\hline RRT & $50(6.3 \%)$ & $24(26.4 \%)$ & $26(3.7 \%)$ & $<0.001$ & \\
\hline Vasopressor use/septic shock & $222(28.0 \%)$ & $65(71.4 \%)$ & $157(22.4 \%)$ & $<0.001$ & $4.96(2.86-8.60) \dagger$ \\
\hline \multicolumn{6}{|l|}{ Microbiology } \\
\hline Invasive pneumococcal disease & $106(13.4 \%)$ & $26(28.6 \%)$ & $80(11.4 \%)$ & $<0.001$ & \\
\hline Positive UAT & $122(15.4 \%)$ & $33(36.3 \%)$ & $89(12.7 \%)$ & $<0.001$ & $2.57(1.49-4.43) \dagger$ \\
\hline \multicolumn{6}{|l|}{ Outcomes } \\
\hline Hospital LOS (days) & $5(2-11)$ & $5(2-14)$ & $5(3-10)$ & 0.873 & \\
\hline Hospital mortality & 89 (11.2\%) & $80(87.9 \%)$ & $9(1.3 \%)$ & $<0.001$ & \\
\hline
\end{tabular}

Abbreviations: $95 \% \mathrm{Cl}=95 \%$ confidence interval; $\mathrm{AED}=$ accident and emergency department; $\mathrm{CNS}=$ central nervous system; $\mathrm{COPD}=$ chronic obstructive pulmonary disease; ECMO = extracorporeal membrane oxygenation; IQR = interquartile range; LOS = length of stay; MSK = musculoskeletal; $\mathrm{RRT}=$ renal replacement therapy defined as intermittent and continuous replacement; vasopressor = dopamine, adrenaline, or noradrenaline; UAT = urinary antigen test

* Data are shown as median (IQR) or No. (\%), unless otherwise specified. Hosmer-Lemeshow goodness-of-fit test was performed for logistic regression analysis $\left(\chi^{2}=6.433\right.$; degrees of freedom $\left.=7 ; P=0.490\right)$

$+\mathrm{P}<0.05$ in logistic regression analysis 


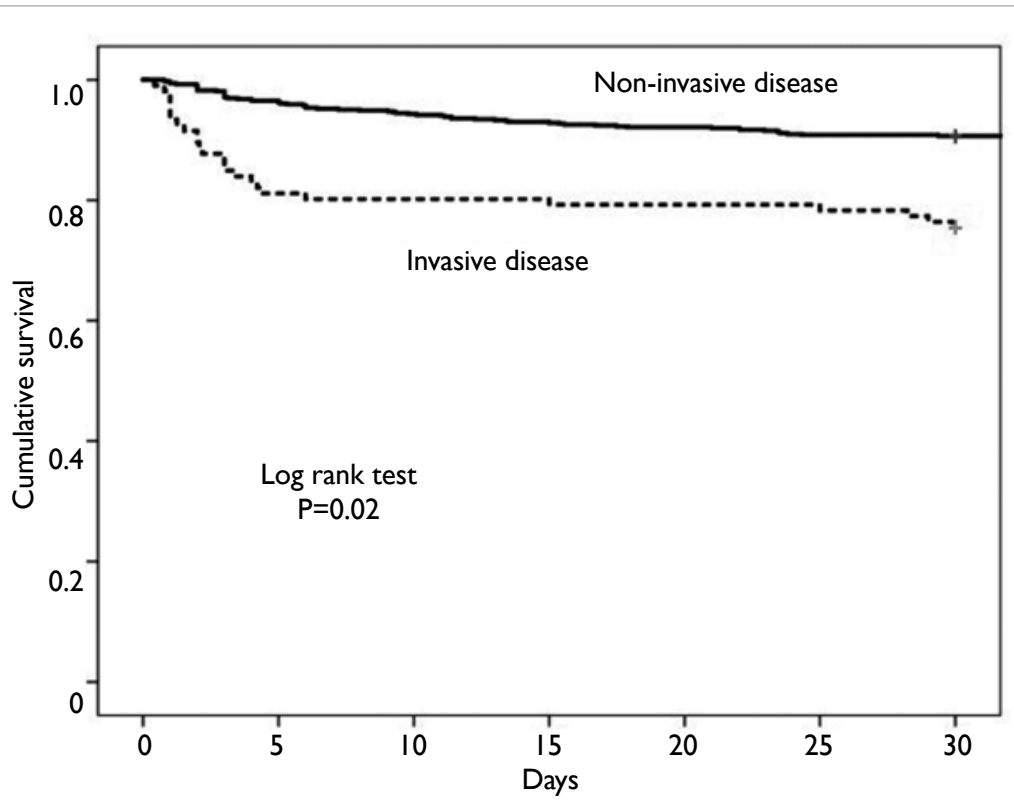

FIG. Kaplan-Meier survival plot of invasive pneumococcal disease lung conditions, which may have affected mortality in these patients.

\section{Pneumococcal urinary antigen test}

In our cohort, 122 patients were diagnosed with pneumococcal infection by using the UAT. In our hospital, the first patient was diagnosed by using the UAT in 2015. Use of the UAT in diagnosing community-acquired pneumonia has since increased; thus, in 2018, 71 of 146 patients (48.6\%) were diagnosed by using the UAT. A positive UAT result was a consistent independent predictor for 30-day mortality among patients in the ICU, as well as among all patients. Post-hoc analysis showed that a positive UAT result was significantly associated with ICU admission $(34.7 \%$ vs $10.1 \%, \mathrm{P}<0.001)$. However, it was not significantly associated with ICU LOS (6.16 vs 8.43 days, $\mathrm{P}=0.515$ ) or hospital LOS (21.46 vs 29.78 days, $\mathrm{P}=0.415$ ).

The pneumococcal UAT assay detects the C-polysaccharide antigen of $S$ pneumoniae, which is present in all serotypes, from urine samples. ${ }^{14}$ Fluorescence immunoassay and immunochromatographic test methods provide similar results in terms of diagnosing pneumococcal disease. ${ }^{15}$ While the UAT result remains positive for up to 3 days after initiation of antibiotic treatment, the UAT increases the diagnostic yield of pneumococcal disease relative to the yield of sputum culture of $S$ pneumoniae; notably, the yield of such sputum cultures markedly decreases after initiation of antibiotic treatment. ${ }^{16}$ This test provides a rapid and simple method for diagnosis of patients with suspected $S$ pneumoniae infection; it is particularly helpful in the diagnosis of patients who cannot produce sputum for cultures. The test sensitivity and specificity were approximately $60 \%$ and $99 \%$, respectively. ${ }^{16}$ Because of the high test specificity, the UAT helps to reduce the costs of further diagnostic tests and aids in selection of empirical antibiotic treatment. It is recommended in the Infectious Diseases Society of America/American Thoracic Society guidelines for aiding the rapid identification of pneumococcal disease in adults. ${ }^{17}$ Urinary antigen tests were also found to predict the severity and outcomes of pneumonia. A Korean group found that patients with positive UAT results exhibited greater severity of disease; however, the test results were associated with rates of ICU admission and mortality. ${ }^{14}$

Counterindications for the UAT include recent pneumococcal disease within 3 months; moreover, it may cross-react with antigens from other streptococcal bacteria. ${ }^{16,18}$ Patients with acute kidney injury due to sepsis, as well as those with oliguria or anuria of various aetiologies may not be able to provide urine samples for use in the UAT. 
TABLE 2. Clinical characteristics of patients with invasive and non-invasive pneumococcal disease*

\begin{tabular}{|c|c|c|c|c|c|}
\hline Characteristics & Total $(n=792)$ & Invasive $(n=106)$ & $\begin{array}{c}\text { Non-invasive } \\
(n=686)\end{array}$ & $P$ value & Odds ratio $(95 \% \mathrm{Cl})$ \\
\hline Age (years) & $73(61-81)$ & $72.5(61-81)$ & $73(61-81)$ & 0.678 & \\
\hline Advanced age (>65 years) & $554(69.9 \%)$ & $69(65.1 \%)$ & $485(70.7 \%)$ & 0.241 & \\
\hline Sex & & & & 0.021 & \\
\hline Male & $581(73.4 \%)$ & $68(64.2 \%)$ & $513(74.8 \%)$ & & \\
\hline Female & $211(26.6 \%)$ & $38(35.8 \%)$ & $173(25.2 \%)$ & & \\
\hline Ever smoker & $200(25.3 \%)$ & $11(10.4 \%)$ & $189(27.6 \%)$ & $<0.001$ & $0.35(0.18-0.67) \dagger$ \\
\hline Sources of infection & & & & $<0.001$ & \\
\hline CNS & $3(0.4 \%)$ & $3(2.8 \%)$ & 0 & & \\
\hline MSK & $2(0.3 \%)$ & $2(1.9 \%)$ & 0 & & \\
\hline Respiratory & $761(96.1 \%)$ & $86(81.1 \%)$ & $675(98.4 \%)$ & & \\
\hline Septicaemia & $10(1.3 \%)$ & $10(9.4 \%)$ & 0 & & \\
\hline Skin & $5(0.6 \%)$ & $2(1.9 \%)$ & $3(0.4 \%)$ & & \\
\hline Others & $11(1.4 \%)$ & $3(2.8 \%)$ & $8(1.2 \%)$ & & \\
\hline \multicolumn{6}{|l|}{ Co-morbidities } \\
\hline Heart failure & 31 (3.9\%) & $4(3.8 \%)$ & 27 (3.9\%) & 1.000 & \\
\hline Asthma/COPD & $193(24.4 \%)$ & $11(10.4 \%)$ & $182(26.5 \%)$ & $<0.001$ & \\
\hline Chronic kidney disease & 95 (12.0\%) & $29(27.4 \%)$ & $66(9.6 \%)$ & $<0.001$ & $3.10(1.88-5.13) \dagger$ \\
\hline Hepatic failure/cirrhosis & $10(1.3 \%)$ & 0 & $10(1.5 \%)$ & 0.374 & \\
\hline Haematological malignancy & $18(2.3 \%)$ & $6(5.7 \%)$ & $12(1.7 \%)$ & 0.012 & \\
\hline Metastatic cancer & $2(0.3 \%)$ & 0 & $2(0.3 \%)$ & 1.000 & \\
\hline Chemotherapy & $68(8.6 \%)$ & $9(8.5 \%)$ & $59(8.6 \%)$ & 0.97 & \\
\hline Diabetes mellitus & $131(16.5 \%)$ & $14(13.2 \%)$ & $117(17.1 \%)$ & 0.321 & \\
\hline Immunocompromised status & $9(1.1 \%)$ & $2(1.9 \%)$ & $7(1.0 \%)$ & 0.344 & \\
\hline \multicolumn{6}{|c|}{ Complications and invasive organ support } \\
\hline Intensive care & $170(21.5 \%)$ & $35(33.0 \%)$ & $135(19.7 \%)$ & 0.002 & \\
\hline Pleural effusion/empyema & $40(5.1 \%)$ & $9(8.5 \%)$ & $31(4.5 \%)$ & 0.082 & \\
\hline Invasive mechanical ventilation & $112(14.1 \%)$ & $26(24.5 \%)$ & $86(12.5 \%)$ & 0.001 & \\
\hline ECMO & $13(1.6 \%)$ & $10(9.4 \%)$ & $3(0.4 \%)$ & 0.399 & \\
\hline Viral co-infection & $22(2.8 \%)$ & $1(0.9 \%)$ & $21(3.1 \%)$ & 0.217 & \\
\hline RRT & $50(6.3 \%)$ & $17(16.0 \%)$ & $33(4.8 \%)$ & $<0.001$ & \\
\hline Vasopressor use/septic shock & $222(28.0 \%)$ & $99(93.4 \%)$ & $123(17.9 \%)$ & $<0.001$ & \\
\hline \multicolumn{6}{|l|}{ Outcomes } \\
\hline 30-Day mortality & $91(11.5 \%)$ & $26(24.5 \%)$ & $65(9.5 \%)$ & $<0.001$ & \\
\hline Hospital LOS (days) & $5(2-11)$ & $8(4-18)$ & $4(2-9)$ & $<0.001$ & \\
\hline Hospital mortality & $89(11.2 \%)$ & $26(24.5 \%)$ & $63(9.2 \%)$ & $<0.001$ & \\
\hline
\end{tabular}

Abbreviations: 95\% Cl = 95\% confidence interval; CNS = central nervous system; COPD = chronic obstructive pulmonary disease; ECMO = extracorporeal membrane oxygenation; IQR = interquartile range; LOS = length of stay; MSK = musculoskeletal; RRT = renal replacement therapy defined as intermittent and continuous replacement; vasopressor = dopamine, adrenaline, or noradrenaline

* Data are shown as median (IQR) or No. (\%), unless otherwise specified

$+\mathrm{P}<0.05$ in logistic regression analysis 
TABLE 3. Clinical predictors for 30-day mortality in the intensive care unit subgroup*

\begin{tabular}{|c|c|c|c|c|c|}
\hline Characteristics & Total $(n=170)$ & $\begin{array}{l}\text { 30-Day deaths } \\
(n=50)\end{array}$ & $\begin{array}{c}\text { 30-Day survivors } \\
(n=120)\end{array}$ & $P$ value & Odds ratio $(95 \% \mathrm{Cl})$ \\
\hline Age (years) & 68 (59-78) & $75.5(63-80)$ & 67 (59-78) & 0.015 & \\
\hline Advanced age ( $>65$ years) & 107 (62.9\%) & $36(72.0 \%)$ & $71(59.2 \%)$ & 0.114 & \\
\hline Sex & & & & 0.949 & \\
\hline Male & $115(67.6 \%)$ & $34(68.0 \%)$ & $81(67.5 \%)$ & & \\
\hline Female & $55(32.4 \%)$ & $16(32.0 \%)$ & 39 (32.5\%) & & \\
\hline Ever smoker & $24(14.1 \%)$ & $4(8.0 \%)$ & $20(16.7 \%)$ & 0.156 & \\
\hline Sources of infection & & & & 0.819 & \\
\hline CNS & $3(1.8 \%)$ & $1(2.0 \%)$ & $2(1.7 \%)$ & & \\
\hline Respiratory & 159 (93.5\%) & 47 (94\%) & 112 (93.3\%) & & \\
\hline Septicaemia & $1(0.6 \%)$ & 0 & $1(0.8 \%)$ & & \\
\hline Skin & $2(1.2 \%)$ & 0 & $2(1.7 \%)$ & & \\
\hline Others & $5(2.9 \%)$ & $2(4.0 \%)$ & $3(2.5 \%)$ & & \\
\hline \multicolumn{6}{|l|}{ Co-morbidities } \\
\hline Heart failure & $7(4.1 \%)$ & $2(4.0 \%)$ & $5(4.2 \%)$ & 1.000 & \\
\hline Asthma/COPD & $23(13.5 \%)$ & $3(6.0 \%)$ & $20(16.7 \%)$ & 0.084 & \\
\hline Chronic kidney disease & $71(41.8 \%)$ & 35 (70.0\%) & $36(30.0 \%)$ & $<0.001$ & $4.64(2.09-10.32) \dagger$ \\
\hline Cirrhosis & $3(1.8 \%)$ & 0 & $3(2.5 \%)$ & 0.556 & \\
\hline Haematological malignancy & $10(5.9 \%)$ & $4(8.0 \%)$ & $6(5.0 \%)$ & 0.482 & \\
\hline Metastatic cancer & $2(1.2 \%)$ & 0 & $2(1.7 \%)$ & 1.000 & \\
\hline Chemotherapy & $14(8.2 \%)$ & $3(6.0 \%)$ & $11(9.2 \%)$ & 0.760 & \\
\hline Diabetes mellitus & $39(22.9 \%)$ & $9(18.0 \%)$ & $30(25.0 \%)$ & 0.323 & \\
\hline Immunocompromised status & $9(5.3 \%)$ & $2(4.0 \%)$ & $7(5.8 \%)$ & 1.000 & \\
\hline \multicolumn{6}{|c|}{ Complications and invasive organ support } \\
\hline Intensive care & $35(20.6 \%)$ & $12(24.0 \%)$ & $23(19.2 \%)$ & 0.478 & \\
\hline Pleural effusion/empyema & $16(9.4 \%)$ & $5(10.0 \%)$ & $11(9.2 \%)$ & 1.000 & \\
\hline Invasive mechanical ventilation & $107(62.9 \%)$ & 38 (76.0\%) & $69(57.5 \%)$ & 0.023 & \\
\hline ECMO & $13(7.6 \%)$ & $7(14.0 \%)$ & $6(5.0 \%)$ & 0.044 & \\
\hline RRT & $50(29.4 \%)$ & $24(48.0 \%)$ & $26(21.7 \%)$ & 0.001 & \\
\hline Vasopressor use/septic shock & $155(91.2 \%)$ & $49(98.0 \%)$ & $106(88.3 \%)$ & 0.043 & \\
\hline APACHE IV score & $91(63.75-117.5)$ & $112.00(95.00-144.50)$ & $81.00(59.00-98.75)$ & $<0.001$ & $3.73(1.27-10.90) \dagger$ \\
\hline \multicolumn{6}{|l|}{ Microbiology } \\
\hline Invasive pneumococcal disease & $35(20.6 \%)$ & $12(24.0 \%)$ & $23(19.2 \%)$ & 0.478 & \\
\hline Viral co-infection & $8(4.7 \%)$ & $1(2.0 \%)$ & $7(5.8 \%)$ & 0.439 & \\
\hline Positive UAT & $56(32.9 \%)$ & $22(44.0 \%)$ & $34(28.3 \%)$ & 0.048 & $2.94(1.32-6.55) \dagger$ \\
\hline \multicolumn{6}{|l|}{ Outcomes } \\
\hline Hospital LOS (days) & $14.5(7-29)$ & $10.00(3.00-18.00)$ & $16.50(9.25-35.75)$ & $<0.001$ & \\
\hline Hospital mortality & $53(31.2 \%)$ & $48(96.0 \%)$ & $5(4.2 \%)$ & $<0.001$ & \\
\hline ICU LOS (days) & $4.05(1.89-9.36)$ & $3.75(1.58-8.27)$ & $4.07(1.90-9.56)$ & 0.394 & \\
\hline
\end{tabular}

Abbreviations: $95 \% \mathrm{Cl}=95 \%$ confidence interval; APACHE IV = Acute Physiology and Chronic Health Evaluation (APACHE IV); CNS = central nervous system; $\mathrm{COPD}=$ chronic obstructive pulmonary disease; $\mathrm{ECMO}=$ extracorporeal membrane oxygenation; ICU = intensive care unit; $I Q R=$ interquartile range; $\mathrm{LOS}=$ length of stay; RRT = renal replacement therapy, defined as intermittent and continuous replacement; vasopressor = dopamine, adrenaline, or noradrenaline

* Data are shown as median (IQR) or No. (\%), unless otherwise specified. Hosmer-Lemeshow goodness-of-fit test was performed for logistic regression analysis $\left(\chi^{2}=2.324\right.$; degrees of freedom $\left.=5 ; P=0.803\right)$

$+\mathrm{P}<0.05$ in logistic regression analysis 


\section{Invasive pneumococcal disease}

In our cohort, IPD was associated with a higher 30-day mortality rate; however, this association did not remain statistically significant in logistic regression analysis. Consistent with the results of previous studies, ${ }^{8,12}$ we found that patients with IPD exhibited more severe disease and worse outcomes. Moreover, IPD was associated with higher rates of ICU admission, invasive organ support (ie, vasopressor use), and renal replacement therapy, as well as longer hospital LOS. The findings might be explained by the higher bacterial load in patients with IPD, which may lead to worse outcomes.

Similar to the study by Ceccato et al, ${ }^{8}$ we did not identify a positive relationship between smoking and IPD. Thus far, results regarding the relationship of smoking with IPD have been inconsistent; the association varies according to local smoking prevalence. ${ }^{11}$ With the implementation of effective smoking cessation programmes and corresponding legislation in Hong Kong, approximately 10\% of individuals $>15$ years of age report daily cigarette consumption; this is markedly lower than the rates in other countries. ${ }^{19,20}$ In our study, smoking status information was extracted from patient records stored in the Hospital Authority Clinical Management System and nursing notes; thus, we may have underestimated the number of smokers in this cohort. Other important aspects of smoking (eg, number of pack-years and passive smoking) were not available for inclusion in this analysis.

Chronic kidney disease has been consistently associated with IPD. A large retrospective observational cohort of 36 million adults revealed a risk ratio of 21.67 for development of IPD among patients with chronic kidney disease. ${ }^{21}$ A Japanese registry showed that the relative risk for IPD among patients with chronic kidney disease ranged from 12.4 to $51.3 .^{10}$ Notably, chronic kidney disease was consistently one of the most important predictors for 30-day mortality among all patients $(\mathrm{OR}=3.62$, $\mathrm{P}<0.001)$ and among patients in the ICU $(\mathrm{OR}=4.64$, $\mathrm{P}<0.001)$.

\section{Intensive care subgroup}

Patients with IPD tended to experience a higher rate of complications and require higher rates of invasive organ support. In particular, patients with IPD more frequently exhibited pleural effusion/empyema; they also more frequently required invasive mechanical ventilation, extracorporeal membrane oxygenation, renal replacement therapy, and vasopressor use. Our sample size may not have been sufficiently powered to demonstrate statistically significant results regarding the ICU subgroup; thus, future studies focused specifically on patients in the ICU may be needed. Other aspects of IPD and use of rescue therapies for acute respiratory distress syndrome (eg, prone ventilation, muscle paralytic agents, and inhaled nitrogen oxide) should be investigated in the future.

\section{Drug non-susceptible Streptococcus pneumoniae and viral co-infection}

Penicillin non-susceptible $S$ pneumoniae was not common in the present study; it was only observed in $2.4 \%$ of patients. Non-susceptibility to levofloxacin was observed in $0.9 \%$ of patients. Drug non-susceptible $S$ pneumoniae were not significantly associated with 30-day mortality (penicillin non-susceptible $S$ pneumoniae was present in two non-survivors and 14 survivors, $\mathrm{P}=0.641$; levofloxacin non-susceptible $S$ pneumoniae was present in zero non-survivors and six survivors, $\mathrm{P}=1.000$ ). However, these results should be carefully interpreted, because of the small number of drug non-susceptible $S$ pneumoniae in our cohort. According to a recent study in Hong Kong, the penicillin resistance rate was approximately $7 \%$ and the levofloxacin resistance rate was $0 \%{ }^{22}$

Viral-bacterial interactions have been described with respect to pneumococcal disease. ${ }^{23}$ An epidemiological study regarding the 2009 H1N1 influenza pandemic period showed a significant increase in the number of pneumococcal pneumonia hospitalisations. ${ }^{24}$ However, viral co-infection was not associated with IPD or mortality in our findings. Notably, an age-specific interaction was described between influenza and IPD; specifically, patients aged 5 to 19 years were significantly more frequently affected, compared with other age-groups. ${ }^{24,25}$

\section{Strengths and limitations}

Thus far, this is the first and largest study regarding pneumococcal disease in adults in Hong Kong; it provides clinical and outcome data in both general ward and intensive care subgroups to allow a comprehensive overview of pneumococcal disease in the locality. It is a standard practice in our centre to check urinary antigens and perform blood cultures for nearly all patients with suspected pneumonia to facilitate accurate diagnosis and avoid missed diagnoses. By including data regarding invasive organ support and ICU admission, we were able to identify and describe complications of pneumococcal disease and determine the broader clinical characteristics of affected patients.

However, because of changes in vaccination programmes, the influenza and pneumococcal vaccination statuses were not available for analysis in the current study. Because of the limited number of patients with drug non-susceptible $S$ pneumoniae in the present cohort, further robust analyses regarding antibiotic sensitivity patterns and appropriateness of antimicrobial treatment could 
not be performed. Furthermore, capsular serotypes of $S$ pneumoniae among patients in our cohort were not available for analysis. Future studies focused on capsular subtypes of $S$ pneumoniae will facilitate understanding of pneumococcal disease. Because this was a retrospective study, it was subject to potential confounding factors. Finally, the results of this single-centre study may not be generalisable to other countries with higher prevalences of drug non-susceptible $S$ pneumoniae infection.

\section{Conclusion}

Pneumococcal disease is associated with high rates of morbidity and mortality. In this cohort, vasopressor use, chronic kidney disease, advanced age, and positive UAT results were predictors for 30-day mortality.

\section{Author contributions}

Concept or design: MY Man, HP Shum. Acquisition of data: MY Man, HP Shum. Analysis or interpretation of data: MY Man, HP Shum. Drafting of the manuscript: MY Man, A Wu. Critical revision of the manuscript for important intellectual content: All authors.

All authors had full access to the data, contributed to the study, approved the final version for publication, and take responsibility for its accuracy and integrity.

\section{Conflicts of interest}

All authors have disclosed no conflicts of interest.

\section{Declaration}

The abstract of this study was accepted as an oral presentation at the Annual Scientific Meeting of the Hong Kong Society of Critical Care Medicine on 8 December 2019.

\section{Funding/support}

This research received no specific grant from any funding agency in the public, commercial, or not-for-profit sectors.

\section{Ethics approval}

This study was approval by the Hospital Authority Hong Kong East Cluster Research Ethics Committee (Ref HKECREC2019-065). The requirement for written informed consent was waived.

\section{References}

1. Aliberti S, Cook GS, Babu BL, et al. International prevalence and risk factors evaluation for drug-resistant Streptococcus pneumoniae pneumonia. J Infect 2019;79:300-11.

2. Black RE, Cousens S, Johnson HL, et al. Global, regional, and national causes of child mortality in 2008: a systematic analysis. Lancet 2010;375:1969-87.

3. Wahl B, O'Brien KL, Greenbaum A, et al. Burden of Streptococcus pneumoniae and Haemophilus influenzae type b disease in children in the era of conjugate vaccines: global, regional, and national estimates for 2000-15. Lancet Glob Health 2018;6:e744-57.

4. Drijkoningen JJ, Rohde GG. Pneumococcal infection in adults: burden of disease. Clin Microbiol Infect 2014;20 Suppl 5:45-51.

5. Song JY, Choi JY, Lee JS, et al. Clinical and economic burden of invasive pneumococcal disease in adults: a multicenter hospital-based study. BMC Infect Dis 2013;13:202.

6. Centre for Health Protection, Department of Health, Hong Kong SAR Government. Number of notifiable infectious diseases by month. 2019. Available from: https://www.chp. gov.hk/en/statistics/data/10/26/43/6830.html. Accessed 5 May 2020.

7. Cillóniz $C$, de la Calle $C$, Dominedò $C$, et al. Impact of cefotaxime non-susceptibility on the clinical outcomes of bacteremic pneumococcal pneumonia. J Clin Med 2019;8:1150

8. Ceccato A, Torres A, Cilloniz C, et al. Invasive disease vs urinary antigen-confirmed pneumococcal communityacquired pneumonia. Chest 2017;151:1311-9.

9. Clinical and Laboratory Standards Institute. M100 Performance standards for antimicrobial susceptibility testing. 29th ed. CLSI supplement M100. Available from: https://clsi.org/media/2663/m100ed29_sample.pdf. Accessed 22 Feb 2020.

10. Imai K, Petigara T, Kohn MA, et al. Risk of pneumococcal diseases in adults with underlying medical conditions: a retrospective, cohort study using two Japanese healthcare databases. BMJ Open 2018;8:e018553.

11. Torres A, Blasi F, Dartois N, Akova M. Which individuals are at increased risk of pneumococcal disease and why? Impact of COPD, asthma, smoking, diabetes, and/or chronic heart disease on community-acquired pneumonia and invasive pneumococcal disease. Thorax 2015;70:984-9.

12. Heo JY, Seo YB, Choi WS, et al. Incidence and case fatality rates of community-acquired pneumonia and pneumococcal diseases among Korean adults: catchment population-based analysis. PLoS One 2018;13:e0194598.

13. van Mens SP, van Deursen AM, de Greeff SC, et al. Bacteraemic and non-bacteraemic/urinary antigenpositive pneumococcal community-acquired pneumonia compared. Eur J Clin Microbiol Infect Dis 2015;34:115-22.

14. Kim B, Kim J, Jo YH, et al. Prognostic value of pneumococcal urinary antigen test in community-acquired pneumonia. PLoS One 2018;13:e0200620.

15. Olofsson E, Özenci V, Athlin S. Evaluation of the sofia S. pneumoniae FIA for detection of pneumococcal antigen in patients with bloodstream infection. J Clin Microbiol 2019;57:e01535-18.

16. Molinos L, Zalacain R, Menéndez R, et al. Sensitivity, specificity, and positivity predictors of the pneumococcal urinary antigen test in community-acquired pneumonia. Ann Am Thorac Soc 2015;12:1482-9.

17. Mandell LA, Wunderink RG, Anzueto A, et al. Infectious Diseases Society of America/American Thoracic Society consensus guidelines on the management of communityacquired pneumonia in adults. Clin Infect Dis 2007;44 Suppl 2:S27-72.

18. Blaschke AJ. Interpreting assays for the detection of Streptococcus pneumoniae. Clin Infect Dis 2011;52 Suppl 4: S331-7.

19. Census and Statistics Department, Hong Kong SAR Government. Thematic Household Survey Report- 
Report No. 70-Pattern of smoking. Available from: https://www.censtatd.gov.hk/hkstat/sub/sp 453. jsp?productCode=C0000047. Accessed 5 May 2020.

20. Wang TW, Asman K, Gentzke AS, et al. Tobacco product use among adults-United States, 2017. MMWR Morb Mortal Wkly Rep 2018;67:1225-32.

21. Zhang D, Petigara T, Yang X. Clinical and economic burden of pneumococcal disease in US adults aged 19-64 years with chronic or immunocompromising diseases: an observational database study. BMC Infect Dis 2018;18:436.

22. Chan KC, Ip M, Chong PS, Li AM, Lam HS, Nelson EA. Nasopharyngeal colonisation and antimicrobial resistance of Streptococcus pneumoniae in Hong Kong children younger than 2 years. Hong Kong Med J 2018;24 Suppl 6:4-7.

23. Blasi F, Mantero M, Santus P, Tarsia P. Understanding the burden of pneumococcal disease in adults. Clin Microbiol Infect 2012;18 Suppl 5:7-14.

24. Weinberger DM, Simonsen L, Jordan R, Steiner C, Miller M, Viboud C. Impact of the 2009 influenza pandemic on pneumococcal pneumonia hospitalizations in the United States. J Infect Dis 2012;205:458-65.

25. Chiavenna C, Presanis AM, Charlett A, et al. Estimating age-stratified influenza-associated invasive pneumococcal disease in England: a time-series model based on population surveillance data. PLoS Med 2019;16:e1002829. 